\title{
On the tensile behaviors of a hard chromium coating plated on a steel substrate with periodic subsurface inclusions
}

\author{
Chen-Wu Wu ${ }^{\mathrm{a}, \mathrm{c}, *}$, Ban-Quan Yang ${ }^{\mathrm{a}, \mathrm{b}, \mathrm{c}}$, Guang-Nan Chen ${ }^{\mathrm{a}}$, Kun Zhang ${ }^{\mathrm{a}}$, Jing-Hua Xiao ${ }^{\mathrm{a}}$ \\ ${ }^{a}$ Institute of Mechanics, Chinese Academy of Sciences, No. 15 Beisihuanxi Road, Beijing, 100080, PR China \\ ${ }^{\mathrm{b}}$ Department of Engineering Mechanics, Armored Force Engineering Institute, Beijing, 100072, PR China \\ ${ }^{c}$ Graduate School of the Chinese Academy of Sciences, Beijing, 100080, PR China
}

Received 6 December 2006; accepted in revised form 27 February 2007

Available online 12 March 2007

\begin{abstract}
The tensile behaviors of a hard chromium coating plated on a steel substrate with periodic laser pre-quenched regions have been investigated by experimental and theoretic analysis. In the experiment, three specimens are adopted to study the differences between homogeneous and periodic inhomogeneous substrates as well as between periodic inhomogeneous substrate of relatively softer and stiffer materials. The unique characteristics have been observed in the specimen of periodic inhomogeneous substrate under quasi-static tension loading. With the periodic laser pre-quenched regions being treated as periodic subsurface inclusions (PSI), the unique stress/strain pattern of the specimen is obtained by analytical modeling and FEM analysis, and the mechanisms accounting for the experimental results is preliminarily illustrated.
\end{abstract}

(C) 2007 Elsevier B.V. All rights reserved.

Keywords: Coating; Periodic subsurface inclusions; Tensile behaviors; FEM

\section{Introduction}

Coatings are adopted in a great many structure withstanding wear, corrosion or thermal degradation. Unfortunately, the prospective applications of such coatings are restricted with its premature spalling. Many designs such as gradient coating, multilayer coating as well as duplex coating [1-3], which arranges the performances of the materials at the direction perpendicular to the interface, are developed to enhance the durability of coating. Such designing improve the lifespan of the coating in a large scale, however in many other cases there are more severe conditions including great thermal gradient or subsurface plastic flowing $[4,5]$ etc., or where the performancegradient perpendicular to the interface is hard to be achieved, more designing other than that aforementioned should be developed to prolong the service of the coatings.

\footnotetext{
* Corresponding author. Institute of Mechanics, Chinese Academy of Sciences, No. 15 Beisihuanxi Road, Beijing, 100080, PR China. Tel.: +86 10 62547527 82; fax: +861062545533.

E-mail address: wcw_on@yahoo.com.cn (C.-W. Wu).
}

Recently, a novel structure of a hard coating (chromium) on a ductile substrate (steel), as shown in Fig. 1, has been developed with laser discrete quenching of the substrate prior to plating coating $[6,7]$. To be noted that, in Fig. 1, the pre-quenching of the upper specimen and the under one is dealt with by Nd: YAG laser and $\mathrm{CO}_{2}$ gas laser, respectively, to obtain the different sizes of the pre-quenched region [7] and the chromium coating is electroplated under room temperature, of which the thickness is about $50-200 \mu \mathrm{m}$. Generally speaking, the pre-quenched regions shall provide a relative stiffer support for the hard coating under contact loading therefore improve the durability of the coating. It has been reported that the hardness of the materials within the laser quenched regions shall be increased by $15 \%-20 \%$ in comparison to that quenched by conventional quenching process due to the existence of the large number of dislocations as well as the finer grains [8-10]. Hence, the hardness of the materials of the laser quenched regions shall be about 2 times that of the intermediate regions between two adjacent as-quenched regions, which is expected not to be influenced visibly by the subsequent plating for which a condition of temperature only slightly higher than room 


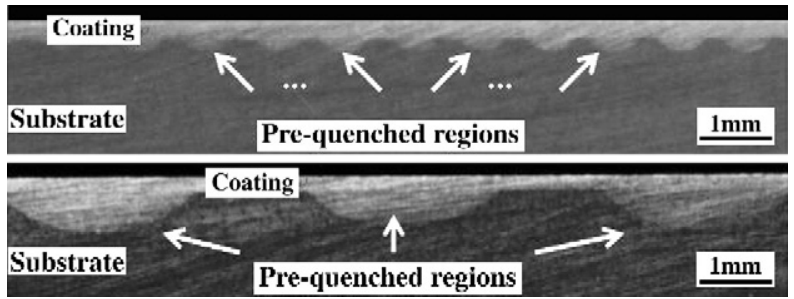

Fig. 1. The cross-section profile of the coating and substrate with periodic prequenched regions [7].

temperature is required $[9,11]$. Moreover, experiments show that the hardness number $H$ is directly proportional to the material's yield stress $Y$, and can be expressed as $H=C Y$, where $C$ is called "constraint factor" and $C=3$ for materials with a large value of the ratio $E / Y$ (e.g., metal), herein $E$ is the elastic modulus $[12,13]$. Therefore, in such a structure, of coating and substrate with periodic laser discrete quenched regions, the yield stress of the surface layer of substrate shall alter periodically along interface. At the same time, laser quenching without melting shall generate residual compressive stresses within the phase transformed regions in contrast to residual tensile stresses within the transition regions between two adjacent phase transformed regions $[8,9,14,15]$. Hence, the mechanical properties and initial stress state shall appear being periodically arranged along interface within the subsurface layer in such a system of coating and substrate. Before now, a classic erosion experiment have been used to investigate the behaviors of the duplex coating under the action of thermal loads and it is found that the surface cracking is partly restrained and the interface cracking is partly arrested [6]. After that, a FEM model was established to investigate the mechanism of the phenomenon of segmentation cracking in the coating when the specimen is subjected to tempering [7] of about $480{ }^{\circ} \mathrm{C}$, of which the periodic pre-quenched regions was treated as periodic subsurface inclusions based on the fact only the eigen-strain due to volume contraction during tempering is taken into account.

In order to attain a more thorough knowledge on such structure, the basic loading conditions, such as tension, bending etc., shall naturally be employed to investigate the corresponding behaviors of the structures. In the present work, a specimen of coating and substrate with periodic laser prequenched regions is directly subjected to tensile loading to study the effect of the periodic laser pre-quenched regions on the tensile behavior of the system. Thereafter, a FEM model is

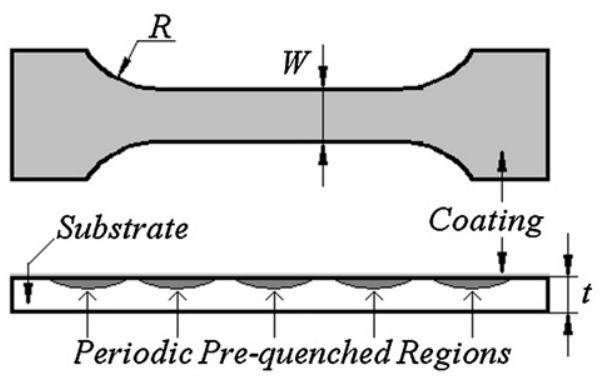

Fig. 2. The sketch of the specimen.
Table 1

The main compositions and mechanical properties of the materials [16-18]

\begin{tabular}{|c|c|c|c|c|}
\hline & Compositions $(\%)$ & $\begin{array}{l}E \\
(\mathrm{GPa})\end{array}$ & $\begin{array}{l}\sigma_{\mathrm{y}} \\
(\mathrm{MPa})\end{array}$ & $\begin{array}{l}\sigma_{\mathrm{f}} \\
(\mathrm{MPa})\end{array}$ \\
\hline A3 & $\begin{array}{l}\mathrm{C} 0.14-0.22, \mathrm{Mn} 0.3-0.65, \mathrm{Si} \leq 0.3, \mathrm{~S} \leq 0.05 \\
\mathrm{P} \leq 0.045\end{array}$ & 200 & 235 & $380-470$ \\
\hline $30 \mathrm{Cr}$ & $\begin{array}{l}\text { C } 0.26-0.34, \text { Si } 0.17-0.37, \text { Mn } 0.3-0.6 \\
\text { Cr } 0.6-0.9, \text { Ni } 2-2.5\end{array}$ & 202 & 784 & 883 \\
\hline \multicolumn{2}{|c|}{ Coating - } & 260 & - & - \\
\hline
\end{tabular}

adopted to investigate the stresses distribution and the cracking behavior of the coating.

\section{The tensile experiment}

Three specimens are prepared with dimensions as shown in Fig. 2 with chromium coating on substrate of homogeneous DIN S235JR steel (NO.1), DIN S235JR steel with periodic laser pre-quenched regions (NO.2) and DIN 28Cr4 steel with periodic laser pre-quenched regions (NO.3), respectively. The former two specimens, NO.1 and NO.2, are used to investigate differences between the behaviors of specimens of homogeneous and periodic inhomogeneous substrate, while the latter two specimens, NO.2 and NO.3, are used to reveal the differences between the behaviors of two substrate materials both with periodic laser pre-quenched regions. To be noted that the as-quenching has been completed with $\mathrm{CO}_{2}$ gas laser. The main compositions and mechanical properties of the two types of substrate materials are listed in Table 1 , where $E, \sigma_{\mathrm{y}}$ and $\sigma_{\mathrm{f}}$ represents, respectively, elastic modulus, yielding stress and uniaxial tensile strength of the material. As for the specimens NO.2 and NO.3, the hardness of surface layer of the substrate materials within a period (herein is about $5 \mathrm{~mm}$ ) is depicted in Fig. 3, of which the HV numbers are measured at the specific locations of distance of $50 \mu \mathrm{m}, 100 \mu \mathrm{m}$ and $150 \mu \mathrm{m}$ from the interface.

The tensile experiment on the three specimens has been completed under the condition of quasi-static state, of which the experimental data, the gauged displacements and the corresponding loading levels, have been recorded. By dividing the

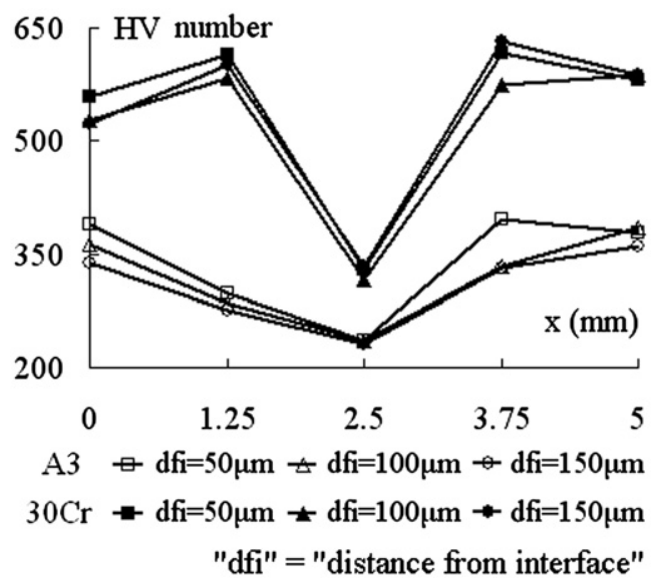

Fig. 3. The HV number of the sub-interface of substrate materials within a period. 


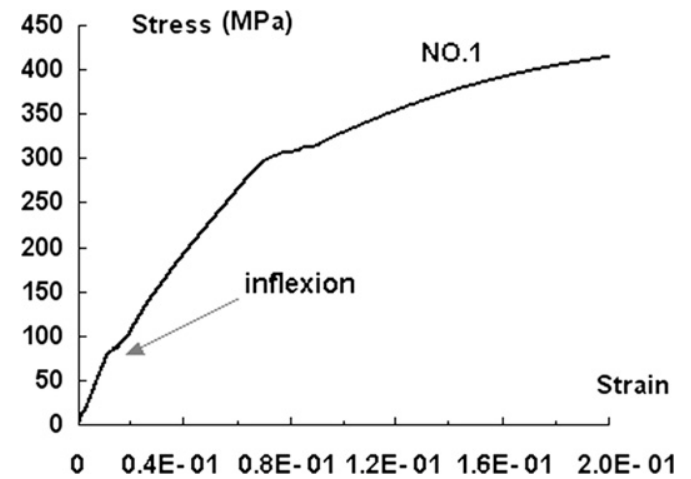

Fig. 4. The nominal stress-strain curve of specimen NO.1.

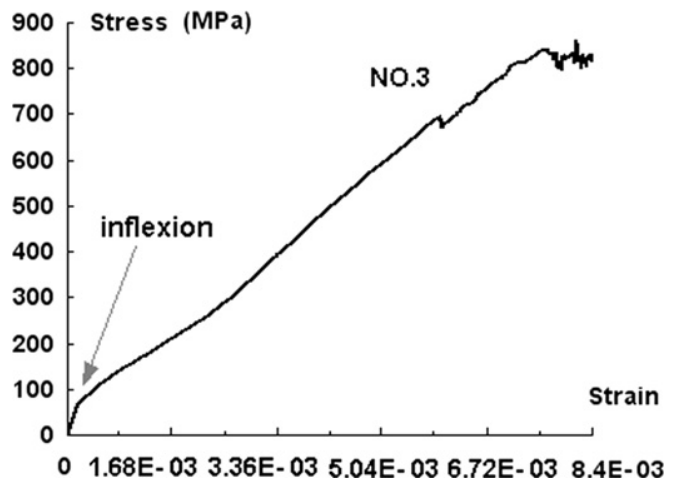

Fig. 6. The nominal stress-strain curve of specimen NO.3. displacement with the gauge distance and the loading with the cross-section area, the nominal strain and stress can be attained and the curves of stress versus strain for the three specimens are plotted in Figs. 4, 5 and 6, corresponding to, respectively, the results from the specimens of NO.1, NO.2 and NO.3. By comparing the curve in Fig. 4 with that in Fig. 5, one can find out, that in the latter, there is seldom flowing stage, while such flowing stage is obvious in the former. Furthermore, the maximum nominal strain is, respectively, $2.0 E-1$ and $2.2 E-2$ before fracture, while the maximum nominal stress is, respectively, $420 \mathrm{MPa}$ and $500 \mathrm{MPa}$. These results show a phenomenological increase in the strength while decrease in the ductility of the system of coating and substrate if the periodic laser discrete pre-quenched regions are introduced into the substrate. This is partly because the pre-quenched regions are with relative higher yielding stress, thus only the local deformation within the finite intermediate regions are possible. Therefore, the overall deformation shall be decreased with the substrate being periodic laser discrete pre-quenched.

By further observing the results in Figs. 4-6, an evident inflexion is found in every stress-strain curve of all three specimens, above which the slopes of the curve decrease. It is indicated that the resultant stiffness of the specimen decreases after the loading above the inflexion. This is may be due to fact that the brittle coating contribute to the resultant stiffness at the early stage, while, above the inflexion, the incremental loading mainly is exerted in the substrate and most of the energy is

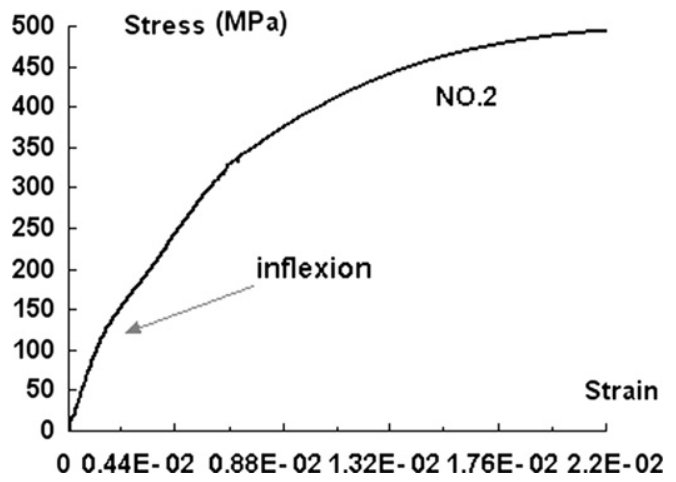

Fig. 5. The nominal stress-strain curve of specimen NO.2. dissipated by the propagations of perpendicular cracks through the coating. Some typical morphologies of the surface and cross section of the fractured specimens are depicted in Figs. 7, 8 and 9, wherein Fig. 7 is corresponding to the specimen of NO.1, Fig. 8 is corresponding to NO.2, and Fig. 9 is corresponding to NO.3, respectively. It is noteworthy that specimens have been etched with the solution of nitric acid with ethanol to present the crosssection profiles in Figs. 8 and 9, of which the dark areas in cross section represent the laser pre-quenched regions in Fig. 8 whereas the bright one represents the laser pre-quenched regions in Fig. 9.

By observing the surface of the specimen NO.1 as shown in Fig. 7, a group of cracks of macroscopically proportional spacing can be found to develop through the coating. Just like the results available in a great many literatures $[19,20]$ on the tensile behaviors of the specimen with brittle coating plated on a homogeneous ductile substrate, the experimental response of NO.1, also, involves the main stages of coating cracking till saturation of cracks, cracks propagating and substrate necking till fracture. However, all of the above characteristics are not so as for the specimen of substrate with periodic pre-quenched regions. The morphology of the specimen NO.2 shown in Fig. 8 indicates that most of the through-coating cracking is confined to the intermediate regions between two pre-quenched regions. Furthermore, multiple quasi-neckings develop within the intermediate regions, which is in particular evident for the relatively softer substrate as depicted in Fig. 8. Herein, the multiple quasinecking, in some sense, can be regarded as the Controlled Diffusion Instability of the extremely tensioned plate, which is implied in [21] as a beneficial characteristic for the formation of

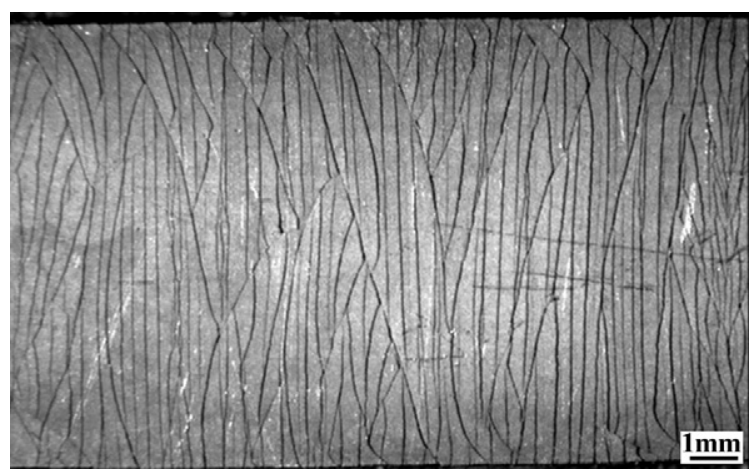

Fig. 7. The surface morphology of specimen NO.1. 


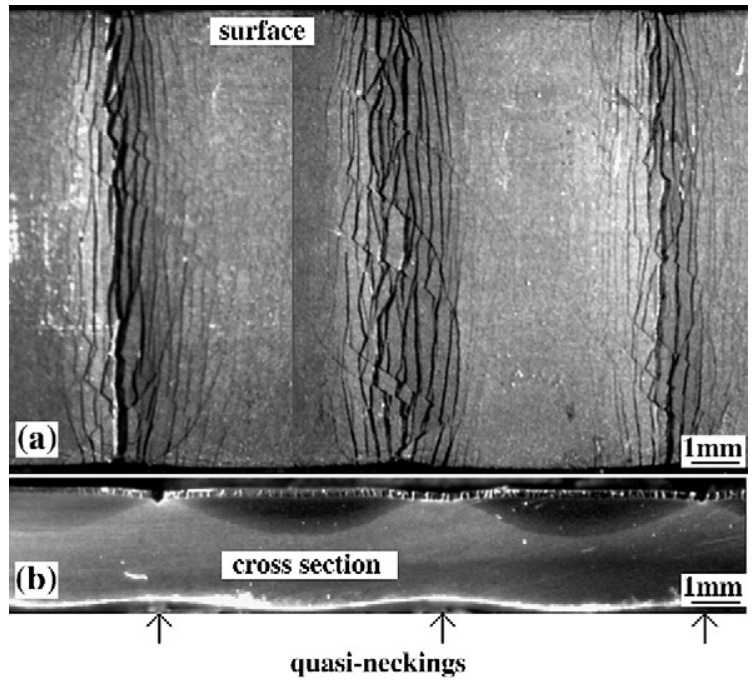

Fig. 8. The surface and cross-section morphologies of specimen NO.2.

thin-walled structure. However, for the present work, partly due to the existence of the brittle coating and its early cracking, the deformation within the multiple quasi-necking regions shall be influenced greatly by the stress concentration around the crack tips. Thus, the beneficial effects of laser treatments on the overall forming performance of the specimen shall be enshrouded.

For the specimen NO.3, with a substrate of a relative stiffer material, the through-coating cracking is still prone to arise within the intermediate regions (refer to Fig. 9(a)). Or even though it can be found other than the intermediate regions, the open displacement of the crack located at the pre-quenched regions is limited to be considerable small (refer to Fig. 9(b)). This implies that the intermediate regions share most of the deformation of the substrate, and hence the pre-quenched regions only experience relatively very small strain, which shall directly lead to a relatively low stress level within the coating bonded to the pre-quenched

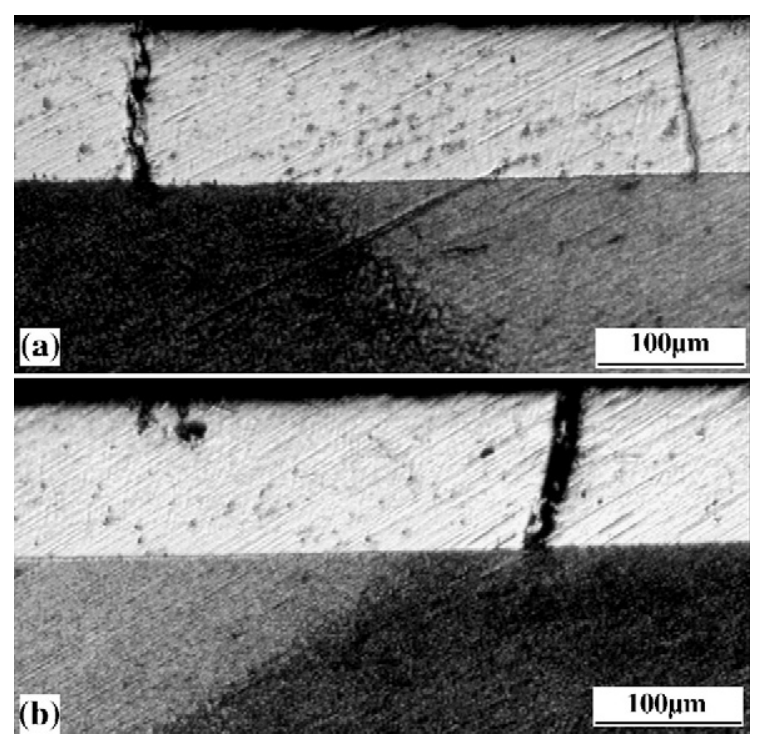

Fig. 9. The cross-section morphology of specimen NO.3.

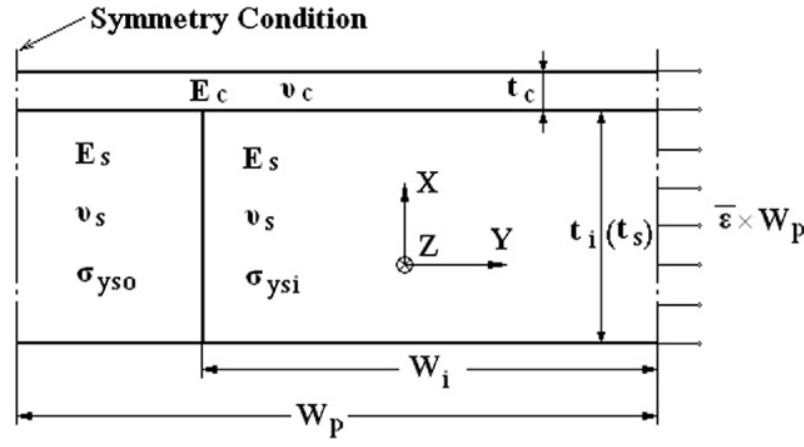

Fig. 10. The analytic model of the specimen with PSI.

regions. To sum up, the specimen of substrate with periodic subsurface inclusions should experience the stages of confined through-coating cracking, multiple quasi-necking, strain localization and final fracture. To inquire into the stress/strain pattern of such specimen of coating plated on substrate with periodic laser pre-quenched regions, analytical modeling and FEM analysis shall be carried through. For the sake of simplification, only the difference in yielding stress between the pre-quenched regions and the origin substrate materials is taken into account without considering the residual stresses within substrate and coating developed in fabrication.

\section{Analytical modeling on the system with PSI}

To investigate the stress distribution of the coating during tension, a simple model of rectangular shape as shown in Fig. 10 is firstly established, with treating the periodic prequenched regions as periodic subsurface inclusions (PSI). Due to the large thickness of the inclusion $\left(t_{\mathrm{i}}=1 \mathrm{~mm}\right)$ relative to that of the coating $\left(t_{\mathrm{c}}=50-200 \mu \mathrm{m}\right)$, the influence of the substrate under the inclusion on deformation of the coating should be slight. Thus such two-layered model is adopted in this preliminary study. Herein the definition of the inclusions means that the elasticity of the inclusions is identical to that of the substrate except for the yielding stress, for the thorough interpretation of which one can refer to [22]. In Fig. 10, $E_{\mathrm{c}}, v_{\mathrm{c}}$, $E_{\mathrm{S}}, v_{\mathrm{s}}$ represent, respectively, the elastic modulus of coating, Possion's ratio of coating, elastic modulus of substrate, and Possion's ratio of substrate. $\sigma_{\mathrm{ys}}, \sigma_{\mathrm{yi}}$ denote, respectively, the

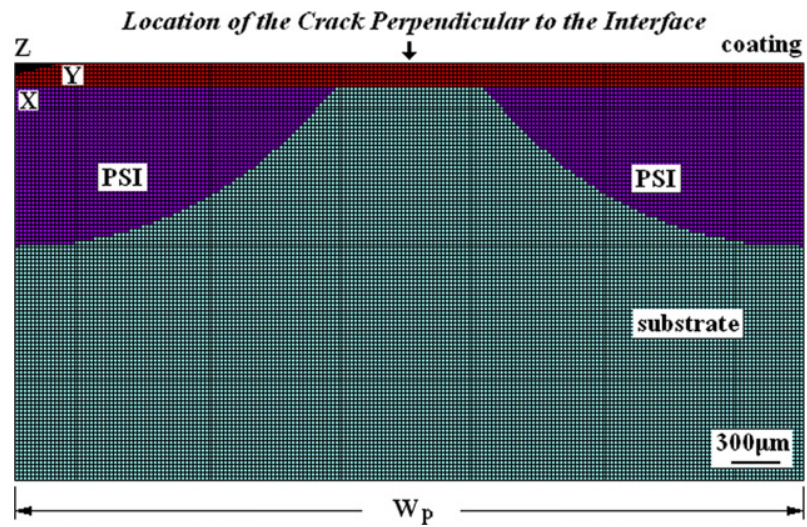

Fig. 11. The sketch of the FEM model and the mesh. 


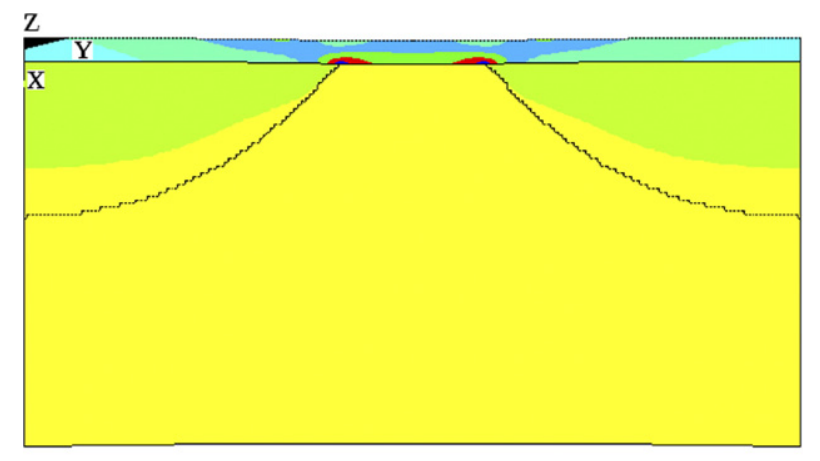

MIN

Fig. 12. The normalized tensile stress of the intact coating

yield stress of the original substrate material, i.e. of the intermediate region and that of the pre-quenched region, i.e. of the periodic subsurface inclusion. It is noteworthy that the elastic perfect-plastic model shall be adopted for both the origin substrate and the inclusion, while the behavior of the hard coating is considered as perfect elastic. Obviously, $\sigma_{\mathrm{ys}}<\sigma_{\mathrm{yi}}$ holds according to the above descriptions. The symbols $t_{\mathrm{c}}, t_{\mathrm{s}}$ represent the thickness of the coating and substrate, respectively, and herein $t_{\mathrm{s}}=t_{\mathrm{i}}$ according to the simplification on modeling. $W_{\mathrm{p}}$ denotes the period width of the structure, herein a period means a cell as shown in Fig. 10 involving an inclusion and an intermediate space between two adjacent inclusions. $W_{\mathrm{i}}$ is the width of the inclusion, i.e. of the laser pre-quenched region. Again we have, $W_{\mathrm{i}} \leq W_{\mathrm{p}}$ according to the fact of discrete quenching, i.e. quenching without overlapping.

Based on the plane strain condition, we shall have $\varepsilon_{z}=0$. Furthermore, if the stress in the direction of $X, \sigma_{x}$, is ignored considering the existence of free surface and the finite stress gradient along $X$-direction, one can immediately get $\sigma_{z}=v \sigma_{y}$ and $\sigma_{y}=E \varepsilon_{y} /\left(1-v^{2}\right)$, where $\varepsilon_{y}$ denotes the strain in the direction $Y$ and $E, v$ is the elastic modulus and Possion's ratio of the materials. This is true for both the coating and the substrate. As for the relatively small thickness of the coating, the tensile rigidity of the specimen should be approximately determined by the substrate with being affected slightly by the coating. Hence, the strain distribution within a cell should be determined roughly only by the action of the substrate. This will be computed as follows.

The Mises equivalent stress (this can be referred to any classic literature on plasticity theory), $\sigma_{\mathrm{e}}$, of the substrate before yielding

$\sigma_{\mathrm{e}}=\sigma_{\mathrm{y}} \sqrt{v_{\mathrm{s}}^{2}-v_{\mathrm{s}}+1}=E_{\mathrm{s}} \varepsilon_{y} \sqrt{v_{\mathrm{s}}^{2}-v_{\mathrm{s}}+1} /\left(1-v_{\mathrm{s}}^{2}\right)$

Thus, the strain $\varepsilon_{\mathrm{si}}$ corresponding to the point wherein the material within the intermediate space between two adjacent inclusions begin to yield can be achieved as

$\varepsilon_{\mathrm{si}}=\frac{\sigma_{\mathrm{ys}}}{E_{\mathrm{s}}} \frac{1-v_{\mathrm{s}}^{2}}{\sqrt{v_{\mathrm{s}}^{2}-v+1}}$
So, the tensile stress within the coating bonded to the inclusion should be

$\sigma_{\mathrm{ci}}=\varepsilon_{\mathrm{si}} E_{\mathrm{c}}=\frac{E_{\mathrm{c}}}{E_{\mathrm{s}}}\left[\sigma_{\mathrm{ys}} \frac{1-v_{\mathrm{s}}^{2}}{\sqrt{\nu_{\mathrm{s}}^{2}-v_{\mathrm{s}}+1}}\right]$

After the origin substrate has reached to the yielding point, the stress, therefore the strain within the inclusions shall not increase any more. Thus, the rest of the total deformation will be exerted, solely, upon the origin substrate between two adjacent inclusions. The strain $\varepsilon_{\mathrm{so}}$ of the origin substrate can be written as

$\varepsilon_{\mathrm{so}}=\frac{\bar{\varepsilon} \times W_{\mathrm{p}}-\varepsilon_{\mathrm{si}} \times W_{\mathrm{p}}}{W_{\mathrm{p}}-W_{\mathrm{i}}}+\varepsilon_{\mathrm{si}}=\frac{\bar{\varepsilon}-\varepsilon_{\mathrm{si}}}{1-W_{\mathrm{i}} / W_{\mathrm{p}}}+\varepsilon_{\mathrm{si}}$

Therefore, the tensile stress within the coating bonded to the origin substrate should be

$$
\begin{aligned}
\sigma_{\mathrm{co}} & =\varepsilon_{\mathrm{so}} E_{\mathrm{c}}=E_{\mathrm{c}}\left[\frac{\bar{\varepsilon}-\varepsilon_{\mathrm{si}}}{1-W_{\mathrm{i}} / W_{\mathrm{p}}}+\varepsilon_{\mathrm{si}}\right] \\
& =E_{\mathrm{c}}\left[\frac{\bar{\varepsilon}-\left(W_{\mathrm{i}} / W_{\mathrm{p}}\right)\left(\frac{\sigma_{\mathrm{ys}}}{E_{\mathrm{s}}} \frac{1-v_{\mathrm{s}}^{2}}{\sqrt{v_{\mathrm{s}}^{2}-v_{\mathrm{s}}+1}}\right)}{1-W_{\mathrm{i}} / W_{\mathrm{p}}}\right]=\frac{E_{\mathrm{c}} \bar{\varepsilon}-\left(W_{\mathrm{i}} / W_{\mathrm{p}}\right) \sigma_{\mathrm{ci}}}{1-W_{\mathrm{i}} / W_{\mathrm{p}}}
\end{aligned}
$$

The results in Eqs. (2) and (3) indicate that, once the origin material of substrate come into yielding, the strain of the inclusions and hence the stress of the coating bonded to the inclusions shall not increase with the further increase of the external loading. Therefore, the periodic inclusions act as hard inclusions when the origin materials of the regions of substrate other than the inclusions are flowing. It is noteworthy that this conclusion only requires that the yielding stress of the inclusion is higher than that of the original substrate material, no matter what is the certain ratio of one to the other.

Nevertheless, a regular figure has been adopted in the above analyzing to discuss the effect of the inclusions on the stress distribution in the coating. Such simple regular model can only

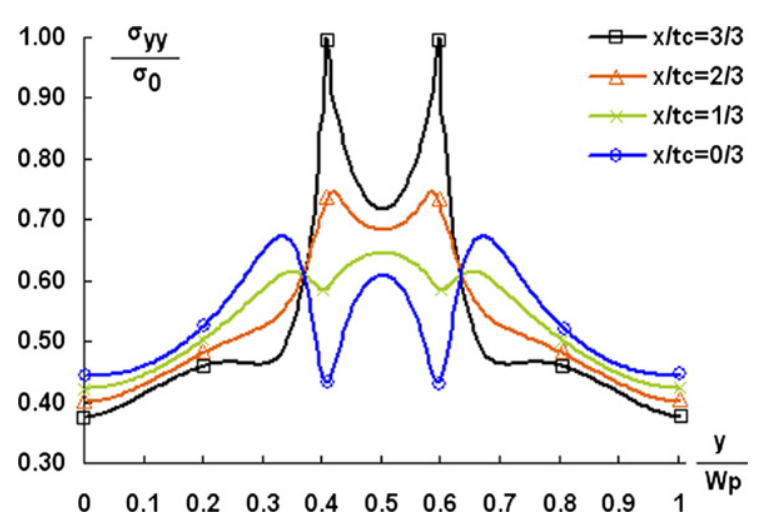

Fig. 13. The contour of the tensile stress of the specimen with intact coating. 


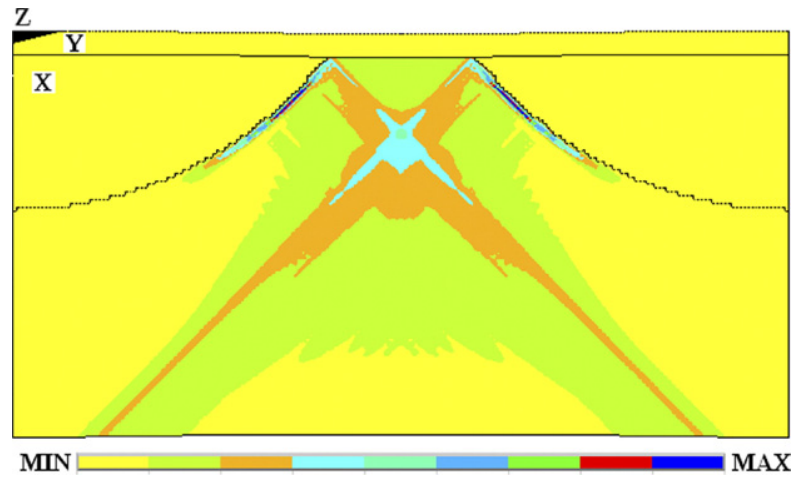

Fig. 14. The plastic strain in $Y$-direction of the specimen with intact coating.

characterize the stress state far away from the interface corner among the coating, origin substrate and the inclusion, wherein the stress state is expected to be considerably complex. To attain a more acceptable description on the stress state and the deformation, a FEM model is established and the computation results are demonstrated as follows.

\section{The FEM computations}

In a cell of width $W_{\mathrm{p}}$, the length of the period of the structure, is modeled as shown in Fig. 11, in which the rectangular Cartesian Coordinates are adopted with the origin located at the surface of the coating above the center of an inclusion. And the mechanical properties of the relatively soft substrate material, DIN S235JR steel, are used in simulation. Firstly, the specimen with an intact coating is modeled and the stress distribution under uniformly tension is computed. The contour of stress in $Y$ direction of coating is shown in Fig. 12, which indicates a prominent stress concentration in the intermediate region. Particularly, high stress level arises around the corner shared by the coating, inclusion and intermediate region as shown in Fig. 12. To describe the stress pattern in coating more clearly, the stresses along a group of paths parallel to the surface are depicted in Fig. 13, in which the symbol $\sigma_{0}=\max \left\{\sigma_{\text {yy }}\right\}$ denotes the peak value of the stress in coating. In Fig. 13, the four curves represent, respectively, the stresses along the path of distance $(0 / 3) t_{\mathrm{c}},(1 / 3) t_{\mathrm{c}},(2 / 3) t_{\mathrm{c}}$ and $(3 / 3) t_{\mathrm{c}}$ apart from the surface. It can be found that the peak stresses arise within the coating bonded to the intermediate regions, and within which

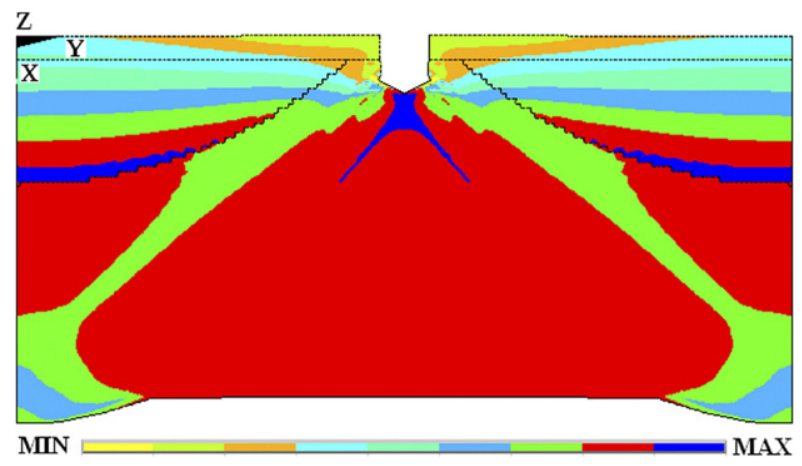

Fig. 15. The normalized tensile stress of the cracked coating.

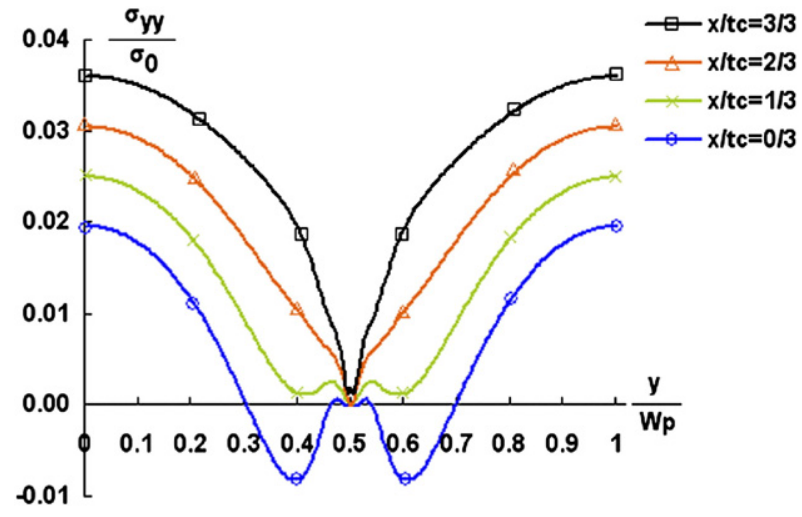

Fig. 16. The contour of the tensile stress of the specimen with cracked coating.

the fluctuation in stresses appears due to the complexity of geometry and performances variation. Fig. 14 displays the plastic strain in $Y$-direction, which shows that the plastic strain of the substrate mainly arises within the intermediate regions between two adjacent inclusions. And, just like that have been expected by the analytic modeling in the previous section, there is no plastic strain within the inclusions. It can be expected herein that the relatively small degree of deformation of the coating in the area above the laser quenched zone shall directly restrict its cracking, which has been validated with the experimental results shown in Figs. 8 and 9.

Now that the stress concentration arise within the intermediate regions as demonstrated in Figs. 12 and 13, and the fact that coating cracking is confined within the intermediate regions according to the experimental results, a coating crack, of length $2 t_{\mathrm{c}}$, located at the mid point of the intermediate region is introduced to the subsequent computation as also figured in Fig. 11. The same tensile loading as used in the previous computation is exerted, and the results corresponding to that in Figs. 12, 13 and 14 are shown in Figs. 15, 16 and 17. In comparing Figs. 12 and 15, one can find that the coating cracking releases the stress of the coating greatly, which can be recognized more clearly in Fig. 16. In Fig. 16, the same paths as that in Fig. 13 are adopted to investigate the stress fluctuation, which indicates that the maximum stress has decreased to less than $4 \%$ of that of the previous case with intact coating. And slight compressive stresses arise within the coating segment around the crack surfaces due to the bending effect. Hence, it

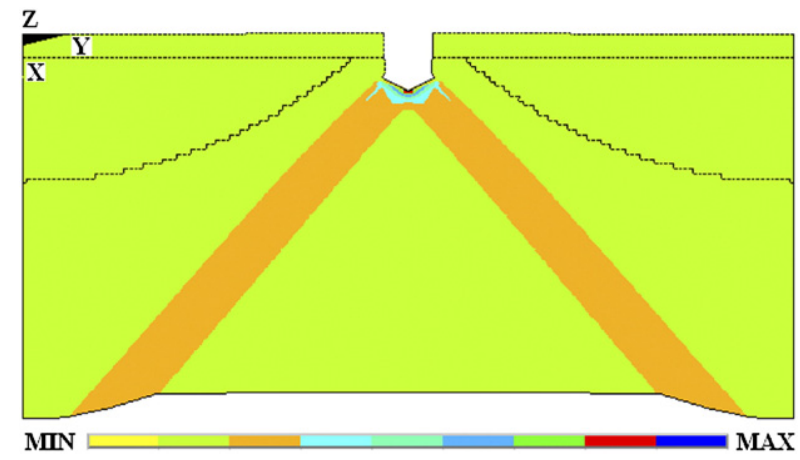

Fig. 17. The plastic strain in $Y$-direction of the specimen with cracked coating 
can be deduced that the cracking of the coating bonded to the intermediate regions shall release most of the stress within the coating and decrease further the driving force of the cracking of the coating bonded to the inclusions. Moreover, the plastic strain shown in Fig. 17 is included within two relatively small declined zonals due to the existence of the crack, in comparison with the large irregular regions as shown in Fig. 14. Again, there is seldom plastic strain outside this declined zonals. And the large necking-alike deformation can be obviously observed in Figs. 15 and 17, of which the large transverse contraction in $X$-direction within the intermediate region is evident, this coincides with the experimental results shown in Fig. 8. As for the cyclic symmetry of the structure, such transverse contraction shall develop within every intermediate region, which is the very multiple quasi-necking aforementioned.

It is worthwhile to note that, though the initial stresses that arise in laser as-quenching have not been involved in both the analytic modeling and the FEM model, some qualitative discussions can be made about the effect of such initial stresses on the tensile behaviors of the specimens. It is accepted that after the literatures $[8,9,14,15]$, laser quenching without melting shall generate residual compressive stresses within the phase transformed regions (corresponding to the PSIs) in contrast to residual tensile stresses within the transition regions (corresponding to the intermediate region) between two adjacent phase transformed regions. One can easily understand that, the initial compressive stress within the PSI shall partly counteract the tensile stress while the initial tensile stress within the intermediate region enhances the tensile stress during tension. Thus, the contribution of such initial stress to the unique behaviors of the tensioned specimens will enhance the so-called confined coating cracking and multiple quasi-necking.

\section{Conclusions}

Phenomenologically, the periodic laser discrete prequenched substrate should increase in the strength while decrease in the ductility of the system of coating and substrate according to the tensile experiment.

The peak stress arises within the coating bonded to the intermediate regions, and hence the coating cracking is confined to the intermediate regions. Once the coating cracking has taken place, the stress shall decrease to a relatively low level all throughout the coating and most of the strain energy of the coating will be dissipated, therefore the possibility of the cracking of the coating bonded to the inclusions will be further decreased.

Due to the fact that the periodic pre-quenched regions act as hard inclusions when the other regions of the substrate come into yielding, multiple quasi-neckings shall arise in the specimen of substrate with periodic pre-quenched regions. This phenomenon is obvious particularly for the relatively soft substrate, say of DIN S235JR steel, which is evidently validated with the experimental and FEM simulation. Hence, the specimen of substrate with periodic subsurface inclusions should experience the stages of confined coating cracking, multiple quasi-necking, strain localization and fracture under the tensile loading.

\section{Acknowledgements}

The financial support provided by the National Natural Science Foundation of China (Grant Nos. 50,471,087, 50,531,060 and $10,572,140)$ is greatly acknowledged. Thanks are also due to the other researchers from the Lab for Surface Modification, Institute of Mechanics, Chinese Academy of Sciences for their help.

\section{References}

[1] D. Kim, M. Kim, K.S. Nam, D. Chang, S.C. Kwon, Surf. Coat. Technol. 169-170 (2003) 650.

[2] C. Pfohl, K.-T. Rie, Surf. Coat. Technol. 142-144 (2001) 1116.

[3] T. Bell, H. Dong, Y. Sun, Tribol. Int. 31 (1998) 127.

[4] J.H. Underwood, M.D. Witherell, S. Sopok, J.C. McNeil, C.P. Mulligan, G.N. Vigilante, Wear 257 (2004) 992.

[5] Herzl Chai, Int. J. Solids Struct. 40 (2003) 6023.

[6] Kun Zhang, Chen-Wu Wu, Ying Hu, Guang-Nan Chen, Solid State Phenom. 118 (2006) 243.

[7] Chen-Wu Wu, Guang-Nan Chen, Kun Zhang, Ying Hu, Nai-Gang Liang, Surf. Coat. Technol. 201 (2006) 2364.

[8] Hengde Li, Jimei Xiao (Eds.), Surfaces and Interfaces of Materials, Tsinghua University Press, Beijing (China), 1990, p. 47, (In Chinese).

[9] Jingxin Qu, Honghong Wang (editors), Handbook for Surface Engineering (In Chinese), Chemical Industry Press, Beijing (China), (1990), p. 529, p.139.

[10] Cai Qingkui, Zhang Xiaowen, Fu Shuyun, Wang Weiguo, J. Northeast. Univ. Technol. 52 (3) (1987) 331 (In Chinese).

[11] Zixun Huang, Chunsu Wu, Theory of Electroplating, China AgriculturalMachine Press, Beijing (China), 1982, p. 1, (In Chinese).

[12] Anthony C. Fischer-Cripps, Nanoindentation, Springer, 2002, p. 8.

[13] Y.T. Cheng, C.M. Cheng, Int. J. Solids Struct. 36 (1999) 1231.

[14] D. Grevey, L. Maiffredy, A.B. Vannes, J. Mech. Work. Technol. 16 (1) (1988) 65.

[15] Abdelkarim Ben Rhima, Jamel Bessrour, Mahmoud Bouhafs, Ridha Khadrani, Int. J. Therm. Sci. 42 (2003) 759.

[16] D.X. Chen (Ed.), Handbook of the Mechanical Design, Chemical Industry Press, Beijing (China), 1993, p. 120, (In Chinese).

[17] Z.X. Zhang, Handbook of Enginery Metal materials, Weapon Industry Press, Beijing (China), 1989, p. 451, (In Chinese).

[18] William H. Safranek (Ed.), The Properties of Electrodeposited Metals and Alloys: A Handbook, Elsevier, 1974, p. 39.

[19] B.F. Chena, J. Hwang, G.P. Yu, J.H. Huang, Thin Solid Films 352 (1999) 173.

[20] Lihe Qian, Shijie Zhu, Yutaka Kagawa, Takahiro Kubo, Surf. Coat. Technol. 173 (2003) 178.

[21] H. Shen, G.N. Chen, G.C. Li, Mater. Sci. Eng., A Struct. Mater.: Prop. Microstruct. Process. 219 (1-2) (1996) 156.

[22] Toshio Mura, Micromechanics of Defects in Solids, Martinus Nijhoff Publishers, 1982, p. 2. 